\section{Evaluation of Lung Density and Its Dosimetric Impact on Lung Cancer Radiotherapy: A Simulation Study}

\author{
Raj Verma T. ${ }^{\text {** }}$ Kumar Painuly N. ${ }^{1}$, Prasad Mishra S. ${ }^{2}$, \\ Yoganathan S. A. ${ }^{3}$, Singh N. ${ }^{1}$, Bhatt M. L. B. ${ }^{1}$, Jamal N. ${ }^{1}$
}

\begin{abstract}
Background: The dosimetric parameters required in lung cancer radiation therapy are taken from a homogeneous water phantom; however, during treatment, the expected results are being affected because of its inhomogeneity. Therefore, it becomes necessary to quantify these deviations.

Objective: The present study has been undertaken to find out inter- and intralung density variations and its dosimetric impact on lung cancer radiotherapy using Monte Carlo code FLUKA and PBC algorithms.

Material and Methods: Density of 100 lungs was recorded from their CT images along with age. Then, after PDD calculated by FLUKA MC Code and PBC algorithm for virtual phantom having density $0.2 \mathrm{gm} / \mathrm{cm}^{3}$ and $0.4 \mathrm{gm} / \mathrm{cm}^{3}$ (density range obtained from CT images of 100 lungs) using Co-60 $10 \times 10 \mathrm{~cm}^{2}$ beams were compared.
\end{abstract}

Results: Average left and right lung densities were $0.275 \pm 0.387$ and $0.270 \pm 0.383$ respectively. The deviation in PBC calculated PDD were $(+) 216 \%,(+91 \%),(+) 45 \%$, $(+) 26.88 \%,(+) 14 \%,(-) 1 \%,(+) 2 \%,(-) 0.4 \%,(-) 1 \%,(+) 1 \%,(+) 4 \%,(+) 4.5 \%$ for $0.4 \mathrm{gm} / \mathrm{cm}^{3}$ and $(+) 311 \%,(+) 177 \%,(+) 118 \%,(+) 90.95 \%,(+) 72.23 \%,(+) 55.83 \%$ , (+)38.85\%, (+)28.80\%, (+)21.79\%, (+)15.95\%, (+)1.67\%, (-) 2.13\%, (+)1.27\%, (+) $0.35 \%,(-) 1.79 \%,(-) 2.75 \%$ for $0.2 \mathrm{gm} / \mathrm{cm}^{3}$ density mediums at depths of $1 \mathrm{~mm}$, $2 \mathrm{~mm}, 3 \mathrm{~mm}, 4 \mathrm{~mm}, 5 \mathrm{~mm}, 6 \mathrm{~mm}, 7 \mathrm{~mm}, 8 \mathrm{~mm}, 9 \mathrm{~mm}, 10 \mathrm{~mm}, 15 \mathrm{~mm}, 30 \mathrm{~mm}, 40 \mathrm{~mm}$, $50 \mathrm{~mm}, 80 \mathrm{~mm}$ and $100 \mathrm{~mm}$, respectively.

Conclusion: Large variations in inter- and intra- lung density were recorded. PBC overestimated the dose at air/lung interface as well as inside lung. The results of Monte Carlo simulation can be used to assess the performance of other treatment planning systems used in lung cancer radiotherapy.

\section{Keywords}

PBC, Monte Carlo Code FLUKA, Variation in Lung Density, Virtual Phantom, Computed Tomography.

\section{Introduction}

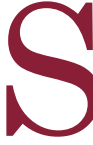
uccess of radiotherapy depends on the accurate dose delivery to the tumor and at the same time minimum dose to normal tissues. To achieve this, knowledge of accurate density of organs coming into the treatment beams is necessary. Human body consists of a variety of tissues and cavities with different physical and radiological properties. Most important among these are tissues and cavities that are radiologically different from water such as lungs and oral cavities. The
${ }^{1}$ King George Medical University, UP, Lucknow, India

${ }^{2}$ Dr.Ram Manohar Lohia Institute of Medical Sciences, Lucknow, India ${ }^{3}$ Sanjay Gandhi Post Graduate Institute of Medical Sciences, Lucknow, India

Corresponding author: T. Raj Verma

Department of Radiotherapy, King George Medical University, UP,

Lucknow; India

E-mail: id-

teerth05kashi@gmail. com

Received: 23 August 2015 Accepted: 28 September 2016 
dose distribution is affected by these tissue inhomogeneities due to difference in density and atomic number compared to water. The presence of inhomogeneity in the path of photon beam will produce increased/decreased transmission depending upon the density and atomic number of inhomogeneity. These decreased/increased photon scatter and the loss of electronic equilibrium result in increased or decreased dose near and inside the inhomogeneity. Lung tissues have different density compared to other organs. Also, lung is one of the most heterogeneous organs in itself. Therefore, it creates more problems as far as lung cancer radiotherapy concerns [1].

A number of methods have been devised to correct these inhomogenieties such as; equivalent tissue air ratio method, isodoe shift, Batho power law, etc. These conventional factorbased algorithms are found not to be able to predict accurate dose distribution in all clinical situations. Several modifications have been incorporated to increase the accuracy of these methods time to time [2-4].

Since its innovation, Computed Tomographic (CT) scanner has been the best tool for density determination. It is the CT data that is used in the treatment planning system (TPSs) for the dose calculation in modern era.

Among current algorithms used in radiotherapy planning, Monte Carlo method is only able to take care of all aspects of interaction of radiation with matter [5]. Along with these features, Monte Carlo simulation code facilitates features of creation of virtual inhomogeneous phantom as found in real practice, and measurement can be performed without measuring equipment. Several Monte Carlo codes are used in radiotherapy treatment planning calculation. In this work, Monte Carlo FLUKA code was used to assess the impact of variation in lung density on dose delivery in lung cancer radiotherapy [6-10].

\section{Aim}

The present study has been undertaken to evaluate the inter- and intra-lung density variations and its dosimetric impact on lung cancer radiotherapy using Monte Carlo code FLUKA and pencil beam convolution (PBC) algorithm.

\section{Material and Methods}

Patients registered in the department of radiotherapy were enrolled in this study. Figure 1 depicts the age distribution of 50 patients included in this study. All enrolled patients had undergone Computed Tomography (CT) scan in the supine position breathing normally as in the normal radiotherapy procedure. The CT scan (Somatom, Siemens Healthcare; US) images of inter slice thickness $3 \mathrm{~mm}$ were taken. These images were stored at the console of CT to evaluate lung density taking three transverse sections one each from the apex, middle and the base of the lung as in Figures 2-4. The same criterion was used for both left and right lungs of each patient. Further, to investigate the intra-lung density variation in the anteriorposterior direction, four planes were selected and average density was calculated using Hounsfield unit as given in equation (1):

$$
\mathrm{D}=1.000+0.001 \mathrm{~N}_{\mathrm{CT}}
$$

Where $\mathrm{D}$ is the electron density $\left(\mathrm{gm} / \mathrm{cm}^{3}\right)$ of lung tissue relative to water and $\mathrm{N}_{\mathrm{CT}}$ is $\mathrm{HU}$

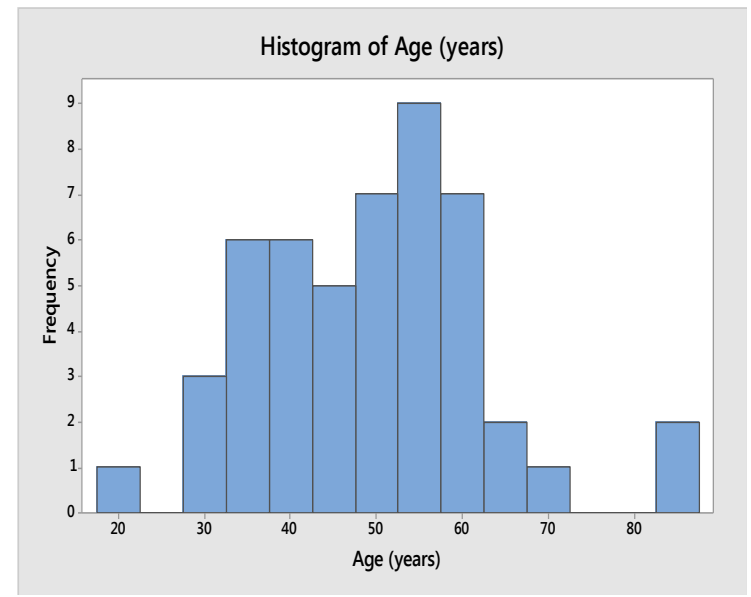

Figure 1: Age Distribution 


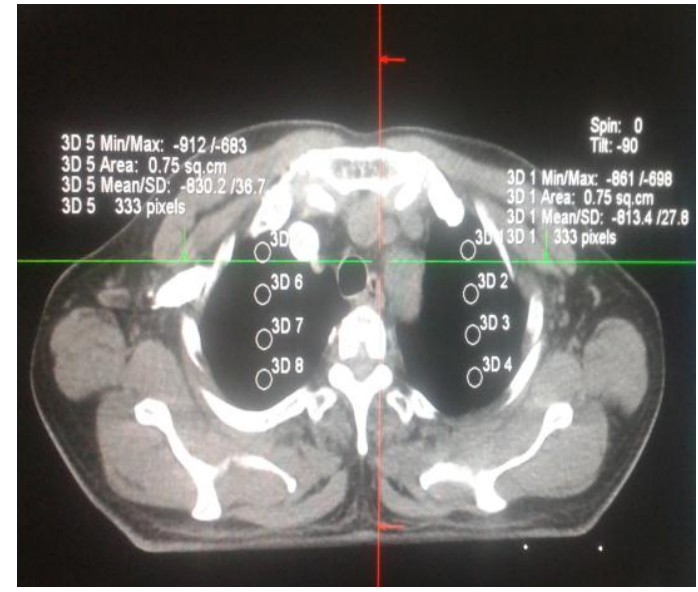

Figure 2: Transverse section of Upper lung along with the four points (circles) to record the intra lung density of right and left lungs

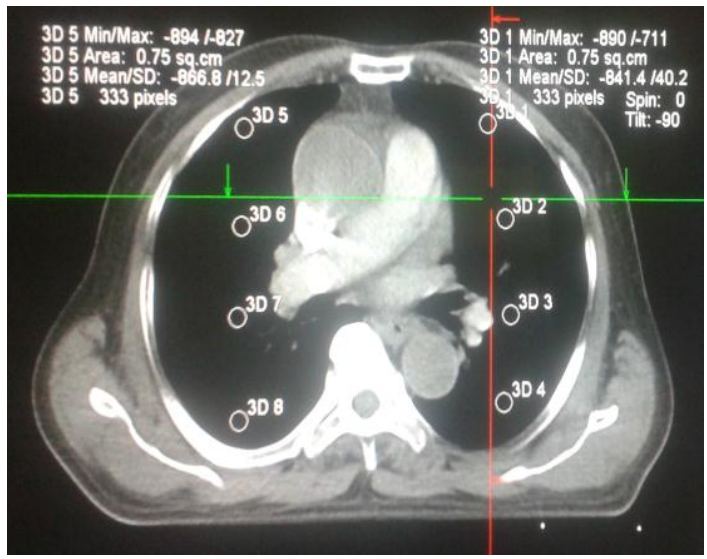

Figure 3: Transverse section of Upper lung along with the four points (circles) to record the intra lung density of right and left lungs

which can be defined in terms of the attenuation coefficients of the material under investigation and water [11].

\section{Monte Carlo Simulation}

For simulation purposes, we have used FLUKA, a general purpose Monte Carlo code capable of handling $\mathrm{KV}$ range to cosmic ray energy. FLUKA code can be used in various fields including medical and accelerator physics. It is widely used for simulation of transportation of particles and interaction of

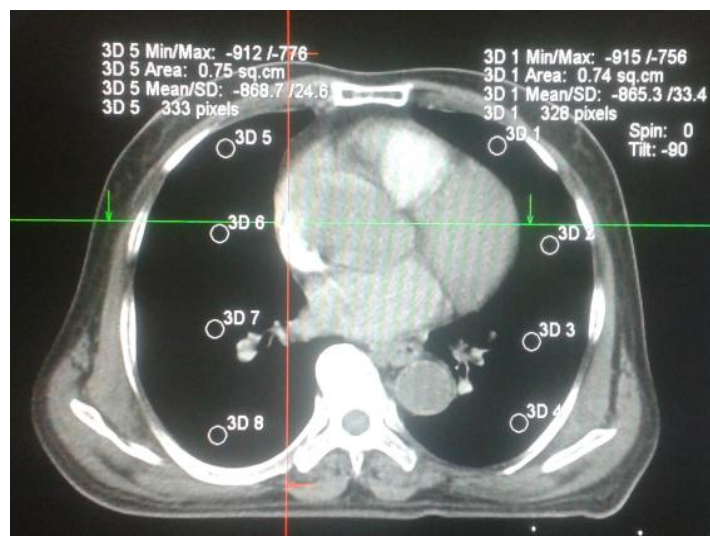

Figure 4: Transverse section of Lower lung along with the four points (circles) to record the intra lung density of right and left lungs

radiation with matter, calorimetry, cosmic ray interaction, radiation protection, detector design, target design dosimetry, space mission, radiobiology, radiotherapy, beam-machine interaction, etc. FLUKA can handle as many as 60 different particles including photon and electron ranging from $\mathrm{Kev}$ to $\mathrm{TeV}$ and can handle very complex geometry using combinational geometry package. FLUKA works on physical models and performs electron transport using multi-scattering approach.

The problem geometry provided in Figure 5 has been incorporated into Monte Carlo code FLUKA. A conical photon beam emitted by Co-60 source at a distance of $80 \mathrm{~cm}$ from detector (phantom) surface has been used in simulation. The average energy of the photon beam emitted from Co-60 source is assumed to be $1.25 \mathrm{MeV}$. The beam is collimated from the starting of the detector surface to a field size of $10 \mathrm{~cm} \times 10 \mathrm{~cm}$. Various materials have been used to fill the phantom of $30 \mathrm{~cm} \times 30 \mathrm{~cm} \times 30$ $\mathrm{cm}$. EM-CASCA default option was selected for the particle transport. USRBIN cards have been used for dose scoring in the phantom at bin size of $1 \mathrm{~mm}$. Number of primary particles has been selected in such a way that relative errors in the estimated doses were $<1 \%$.

The performance of FLUKA code was validated by comparing the PDDs along central 


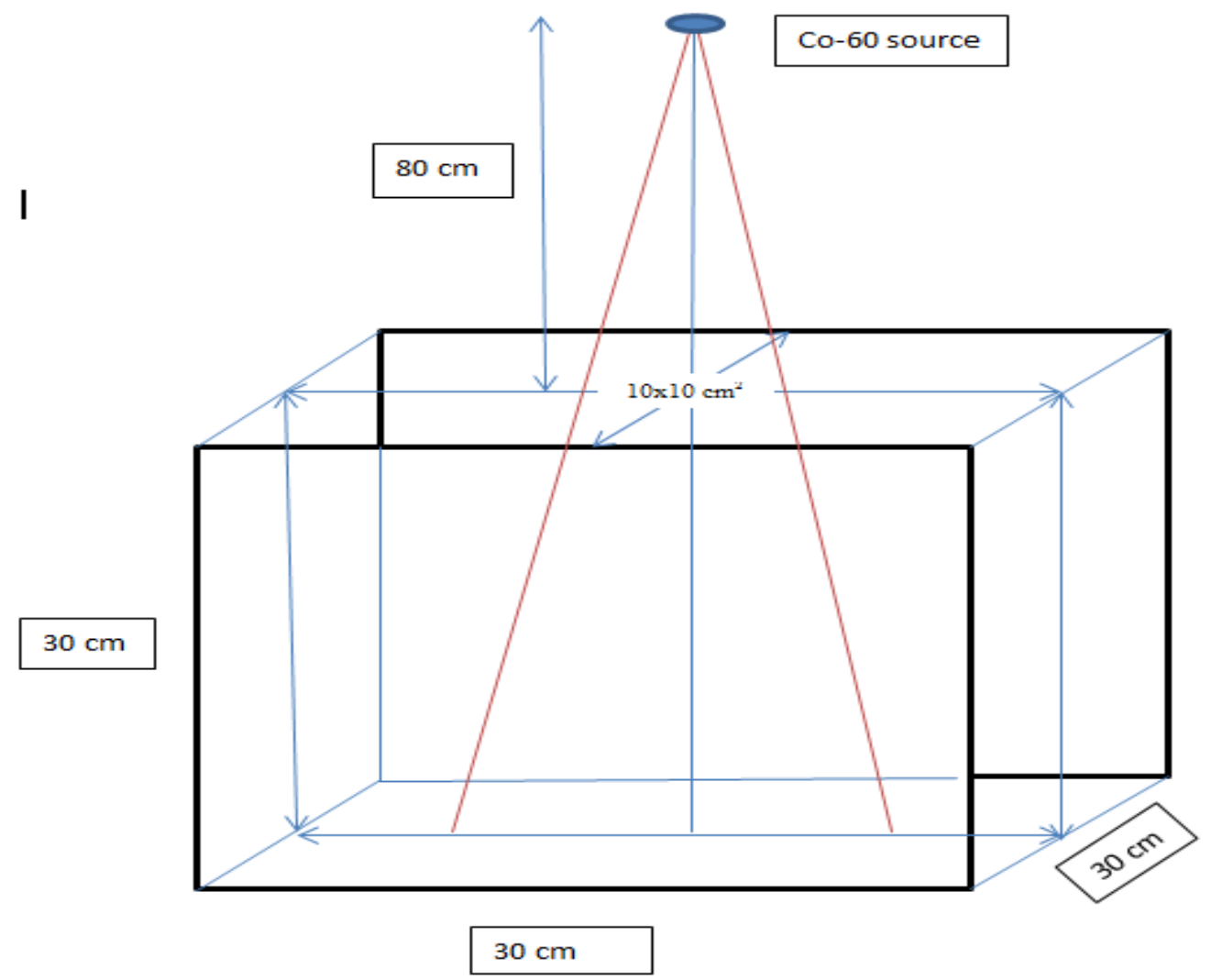

Figure 5: Phantom setup for the calculation of PDD for medium having (1) Water equivalent (2) $0.4 \mathrm{gm} / \mathrm{cm} 3$ (3) $0.2 \mathrm{gm} / \mathrm{cm}^{3}$ density

axis generated from the simulation of $10 \times 10$ $\mathrm{cm}^{2}$ field size of $\mathrm{C} 0-60$ employed to irradiate the $30 \times 30 \times 30 \mathrm{~cm}^{3}$ water phantom and PDD of $10 \times 10 \mathrm{~cm}^{2}$ from BJR supplement 25 data [12]. Simulations were also performed by replacing water with another medium having density 0.2 $\mathrm{gm} / \mathrm{cm}^{3}, 0.27 \mathrm{gm} / \mathrm{cm}^{3}$ and $0.4 \mathrm{gm} / \mathrm{cm}^{3}$, respectively.

\section{TPS Measurement}

To assess the dose calculation accuracy of commercially available PBC algorithm based TPS (Eclipse V 8.6) on variation in lung density. Eclipse TPS (V 8.6) provides the feature of assigning a particular density value to phantom created inside it. To validate the results from TPS, virtual phantom of dimension $30 \times 30 \times 30 \mathrm{~cm}^{3}$ was created assigning water equivalent density and PDD was obtained along the central axis and was compared with standard BJR report. To assess the impact of variation in lung density on PDD, a virtual phantom of dimension $30 \times 30 \times 30 \mathrm{~cm}^{3}$ was created and assigned lung equivalent densities of $0.2 \mathrm{gm} / \mathrm{cm}^{3}$ and $0.4 \mathrm{gm} / \mathrm{cm}^{3}$ that were obtained from 100 lungs using the above-mentioned procedure.

\section{Results}

The variation in lung density with age was found. We have found a relationship between age and respective density of lung. Interestingly, there is slight variation in relationship between age and lung density for left and right lungs for the same patient.

$$
\begin{array}{ll}
Y=-0.0012 x+0.3341 & \text { (left lung) } \\
Y=-0.0015 x+0.3424 & \text { (right lung) }
\end{array}
$$

Figures 6 and 7 demonstrate the variation of lung density with age. Average left lung density for the sample was found $0.275 \pm 0.387$ and 


\section{LEFT LUNG DENSITY Vs AGE}

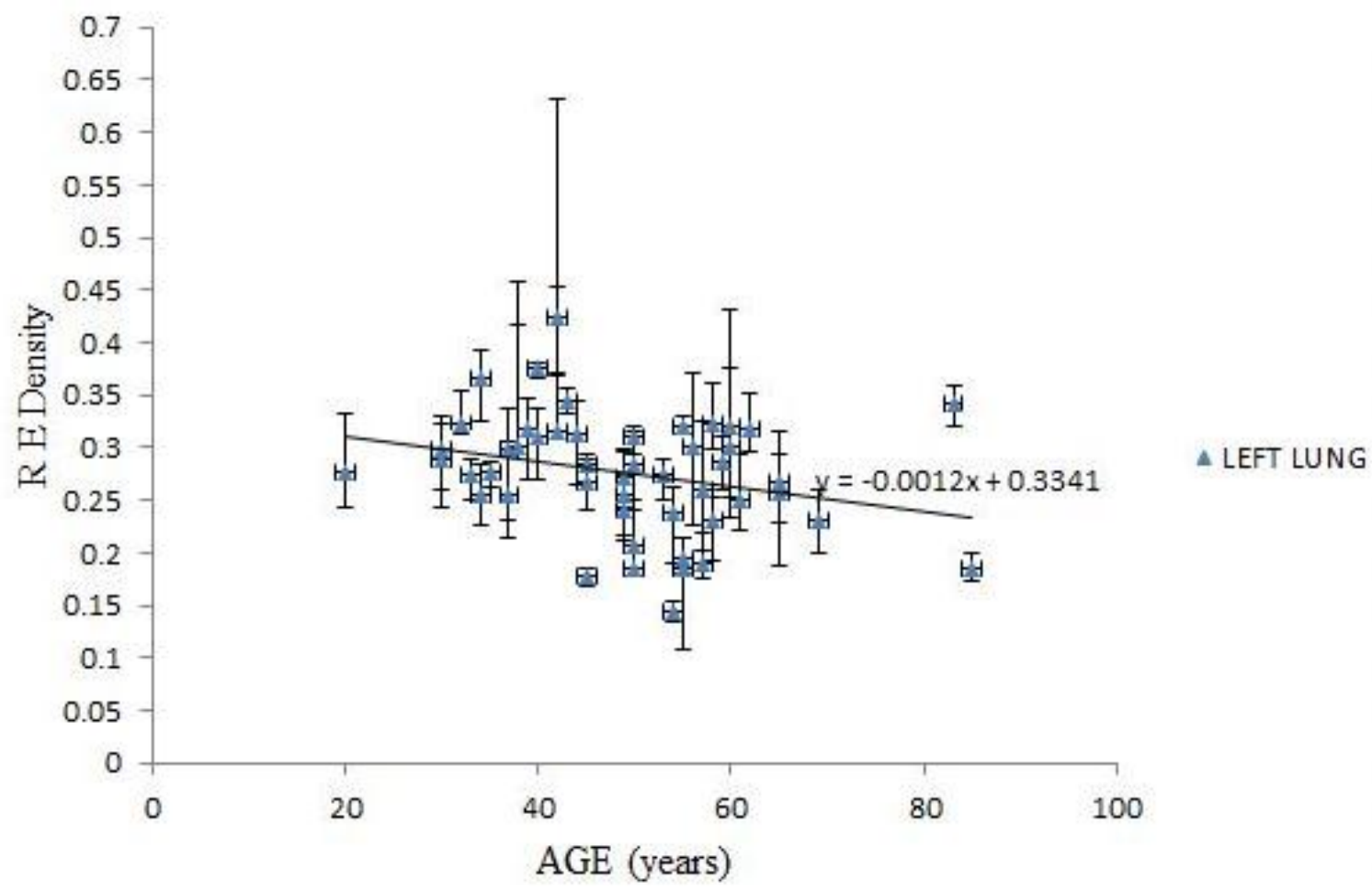

Figure 6: Variation of left lung density with age

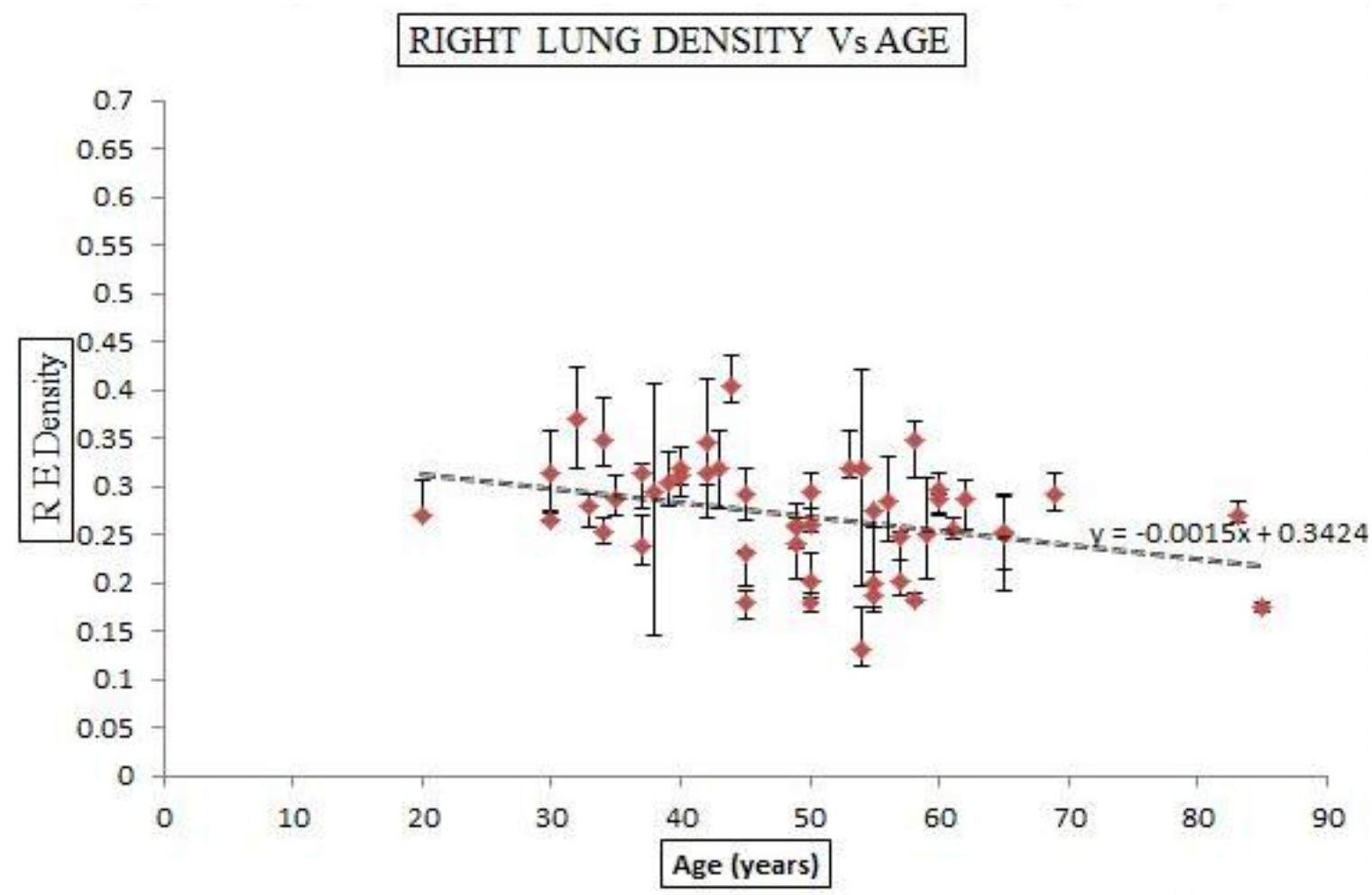

Figure 7: Variation of right lung density with age 
correlation coefficient with age was -0.2765 . Similarly, average right lung density was found $0.270 \pm 0.383$ with correlation coefficient -0.3478 . The correlation coefficient between the density of left and right lung was 0.8258 . From these density data, a relationship between age and lung density was established using the least square fit method [13]. Table 1 contains the calculated lung density using the relationship found from the least square fit method for a particular age.

\section{Validation of TPS and FLUKA Code}

For the validation purposes, FLUKA MC Code derived PDD of water equivalent medium were compared with BJR supplement 25 values. As shown in Table 2, difference of less than $0.5 \%$ was found between two PDDs. Moreover, Pearson's correlation coefficient was found 0.997 for FLUKA PDDs and BJR PDDs values with $p$ value 0.00001 . Similarly, negligible difference between TPS calculated PDD and BJR PDDs value was found with

Table 1: Lung density calculated by least square fit method for different ages.

\begin{tabular}{cc} 
Age & Calculated Lung Density \\
\hline 5 & 0.331 \\
\hline 10 & 0.253 \\
\hline 15 & 0.318 \\
\hline 20 & 0.310 \\
\hline 25 & 0.305 \\
\hline 30 & 0.299 \\
\hline 35 & 0.292 \\
\hline 40 & 0.286 \\
\hline 45 & 0.279 \\
\hline 50 & 0.273 \\
\hline 55 & 0.266 \\
\hline 60 & 0.260 \\
\hline 65 & 0.253 \\
\hline 70 & 0.247 \\
\hline 75 & 0.240 \\
\hline 80 & 0.234
\end{tabular}

Pearsons's correlation coefficient 1.

\section{Comparison of PBC Algorithm and FLUKA MC Code}

The difference in FLUKA calculated PDD for water and lung equivalent medium(s) of density $0.2 \mathrm{gm} / \mathrm{cm}^{3}\left(0.4 \mathrm{gm} / \mathrm{cm}^{3}\right)$ were $21.75 \%$ (16.18\%), 41.54(13.39), 9.64(-3.26), -3.07($0.85),-6.80(-2.44),-9.96(2.39),-8.6(-7.38)$ at $1 \mathrm{~mm}, 5 \mathrm{~mm}, 10 \mathrm{~mm}, 15 \mathrm{~mm}, 20 \mathrm{~mm}, 25 \mathrm{~mm}$, $30 \mathrm{~mm}$ depths (Figure 8).

PDDs for the mediums having density 0.2 $\mathrm{gm} / \mathrm{cm}^{3}$ and $0.4 \mathrm{gm} / \mathrm{cm}^{3}$ were generated for $10 \times 10 \mathrm{~cm}^{2}$ field size using FLUKA code and PBC algorithm-based TPS (Eclipse V 8.6). Significant difference in PDDs from these two was observed. With increase in density, PDD was found decreasing; however, PDDs corresponding to depths less than depth of maximum dose (Dmax) Figure 2 showed an opposite trend. At $5 \mathrm{~mm}$ depth, PDDs were $99.00 \%$ and $86.61 \%$ from PBC algorithm and FLUKA code respectively for medium having density $0.4 \mathrm{gm} / \mathrm{cm}^{3}$. Similarly, for medium having density $0.2 \mathrm{gm} / \mathrm{cm}^{3}$, the PDDs were $100 \%$ and $58.46 \%$ from PBC algorithm and FLUKA code respectively at depth $5 \mathrm{~mm}$. However, opposite

Table 2: Comparison of PDDs generated by MC Code FLUKA with BJR report.

\begin{tabular}{cccc}
\multirow{2}{*}{$\begin{array}{c}\text { Depth (cm) } \\
\text { in Water }\end{array}$} & \multicolumn{2}{c}{ PDD } & \multirow{2}{*}{ Difference } \\
\cline { 2 - 3 } & MC & BJR & \\
\hline 1 & 96.74 & 98.1 & $0.01 \%$ \\
\hline 2 & 93.37 & 93.7 & $-0.35 \%$ \\
\hline 3 & 88.31 & 88.7 & $-0.43 \%$ \\
\hline 4 & 83.55 & 83.7 & $-0.18 \%$ \\
\hline 5 & 78.8 & 78.8 & $0 \%$ \\
\hline 6 & 74.36 & 73.9 & $-0.60 \%$ \\
\hline 7 & 69.70 & 69.3 & $-0.57 \%$ \\
\hline 8 & 64.78 & 64.7 & $0.12 \%$ \\
\hline 9 & 60.53 & 60.5 & $0.00 \%$ \\
\hline 10 & 56.41 & 56.4 & $0.00 \%$
\end{tabular}


Variation of PDD in Mediums of density equivalent to water, $0.2 \& 0.4$ calculated by MC

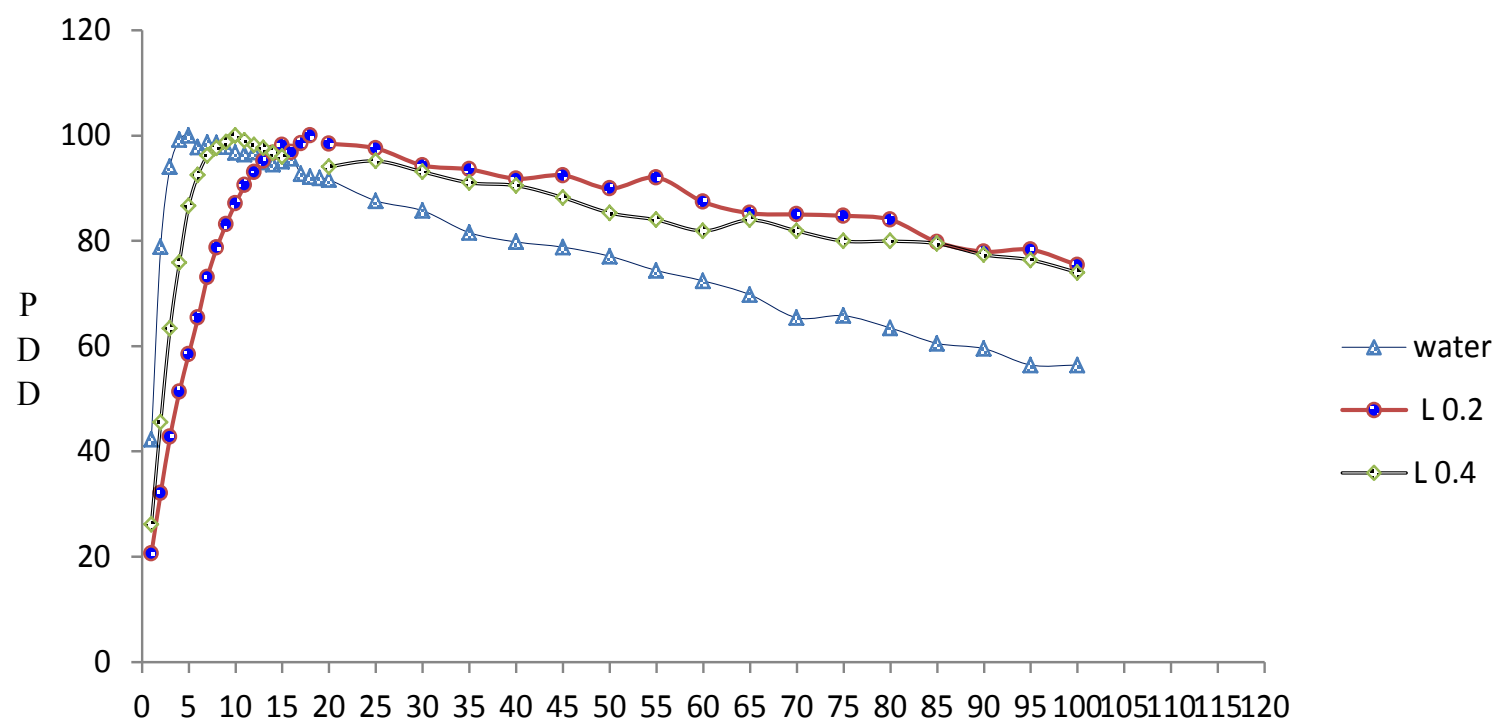

$\operatorname{Depth}(\mathrm{mm})$

Figure 8: FLUKA MC Code generated PDD curve for the set up shown in Figure 6

to this trend, PDDs were $89.92 \%$ and $85.30 \%$ for density $0.2 \mathrm{gm} / \mathrm{cm}^{3}$ and $0.4 \mathrm{gm} / \mathrm{cm}^{3}$ produced by FLUKA MC Code. The percentage deviation in PBC calculated PDD compared to FLUKA code were $(+) 216 \%,(+91 \%),(+) 45 \%$, (+)26.88\%,14\%, $-1 \%, 2 \%,-0.4 \%,-1 \%, 1 \%$, $4 \%, 4.5 \%$ at $1 \mathrm{~mm}, 2 \mathrm{~mm}, 3 \mathrm{~mm}, 4 \mathrm{~mm}, 5 \mathrm{~mm}$, $10 \mathrm{~mm}, 15 \mathrm{~mm}, 30 \mathrm{~mm}, 40 \mathrm{~mm}, 50 \mathrm{~mm}, 80 \mathrm{~mm}$ and $100 \mathrm{~mm}$, respectively in $0.4 \mathrm{gm} / \mathrm{cm}^{3}$. In case of medium having density $0.2 \mathrm{gm} / \mathrm{cm}^{3}$, the percentage deviations were $311 \%, 177 \%$, $118 \%, 90.95 \%, 72.23 \%, 55.83 \%, 38.85 \%$, $28.80 \%, 21.79 \%, 15.95 \%, 1.67 \%,-2.13 \%$, $1.27 \%, 0.35 \%,-1.79 \%,-2.75 \%$ at $1 \mathrm{~mm}, 2 \mathrm{~mm}$, $3 \mathrm{~mm}, 4 \mathrm{~mm}, 5 \mathrm{~mm}, 6 \mathrm{~mm}, 7 \mathrm{~mm}, 8 \mathrm{~mm}, 9 \mathrm{~mm}$ , $10 \mathrm{~mm}, 15 \mathrm{~mm}, 30 \mathrm{~mm}, 40 \mathrm{~mm}, 50 \mathrm{~mm}, 80 \mathrm{~mm}$ and $100 \mathrm{~mm}$, respectively.

\section{Discussion}

From the results, it was found that lung density varies. The range of lung density was found to be 0.45 to 0.2 with average $0.275 \mathrm{gm} /$ $\mathrm{cm}^{3}$. A tendency of decrease in lung density with age was found. Fujisaki T et al. evaluated experimentally and theoretically the impact of change in lung density on dose distribution during lung cancer stereotactic radiotherapy by determining the relative electron density of 30 patients from their CT images, and similar to the present study, a decrease in lung density with age was recorded [14]. To validate the accuracy of $\mathrm{CT}$, quality assurance tests were performed as recommended for clinical purposes and results were under tolerance.

The data used for the calculation of treatment time in manual as well as algorithm-based TPSs for cancer of lung (inhomogeneous in nature) treatment are taken from the measurements done on water phantom (homogenous) [15]. To quantify the impact of this ambiguity between inhomogeneous and homogeneous, authors recorded the PDD for the mediums having density $1 \mathrm{gm} / \mathrm{cm}^{3}$ (water) and $0.20 \mathrm{gm} /$ $\mathrm{cm} 3$ and $0.4 \mathrm{gm} / \mathrm{cm}^{3}$ (lung equivalent).

Change in PDD from $78.80 \%$ to $87.05 \%$ and from $65.78 \%$ to $79.57 \%$ at $5 \mathrm{~cm}$ and 8 $\mathrm{cm}$ depth respectively was found due to the change in density from $1 \mathrm{gm} / \mathrm{cm}^{3}$ to $0.275 \mathrm{gm} /$ 
$\mathrm{cm}^{3}$ for $10 \times 10 \mathrm{~cm}^{2}$ Co-60 beams. RD Praveen kumar et al. conducted a study to estimate the inhomogeneity correction factors for Co-60 beam using Monte Carlo simulation. Results of current study also indicated that inhomogeneity correction factors should be applied in case of manual planning for accurate dose delivery [16].

The variation in density of lung along the path of radiation produces disequilibrium of electron transport. Additionally, the difference in density between body tissue and lung produces the same event. Monte Carlo (modelbased) algorithms have shown the best performance in existing algorithms incorporating all these inhomogeneities [17]. The variation in lung density requires attention not only in manual planning but also in sophisticated TPS that uses algorithms for the dose distribution calculation taking variations in density including lung, different density tissues and bone.

In current study, to demonstrate the dosimetric impact of variation in lung density compared to water (approximately tissue equivalent) as well as variation in lung density itself, phantoms were created of dimension $30 \times 30 \times 30 \mathrm{~cm}^{3}$ (Figure 5) in Monte Carlo simulation code as well as in TPS. Phantoms of $0.4 \mathrm{gm} / \mathrm{cm}^{3}$ density $\& 0.2 \mathrm{gm} / \mathrm{cm}^{3}$ density and tissue equivalent density were created. PDD was recorded from the dose calculated by MC code FLUKA for each density using $10 \times 10 \mathrm{~cm}^{2}$ field size beam of Co-60. Figure 8 demonstrates the PDD Vs Depth for different densities and depicts the fact that as depth of point of interest increases, the difference in PDD increases more rapidly. Table 3 illustrates difference of PDDs of water equivalent medium and medium having lung equivalent density. These differences cause overdosing (especially in manual planning) when not taken into consideration (Figure 9). However, at the surface of inhomogeneity like lung equivalent mediums electronic disequilibrium creates additional source of error [18].

As depicted in Figures 10 and 11, difference between PBC and FLUKA MC was the high-
Table 3: Comparison of PDDs for water equivalent and Lung equivalent mediums generated by MC code FLUKA

\begin{tabular}{cccc}
\multirow{2}{*}{$\begin{array}{c}\text { Depth } \\
\text { (cm) }\end{array}$} & \multicolumn{2}{c}{ PDD } & Difference \\
\cline { 2 - 3 } & $\begin{array}{c}\text { Water } \\
\text { Equivalent }\end{array}$ & $\begin{array}{c}\text { Lung } \\
\text { Equivalent } \\
\text { (0.275gm/ } \\
\mathbf{c m}^{\mathbf{3}} \mathbf{n}\end{array}$ \\
\hline 1 & 96.74 & 91.93 & $-04.97 \%$ \\
\hline 2 & 92.37 & 95.78 & $03.69 \%$ \\
\hline 3 & 87.61 & 93.55 & $08.93 \%$ \\
\hline 4 & 81.55 & 90.65 & $13.31 \%$ \\
\hline 5 & 78.8 & 87.05 & $12.65 \%$ \\
\hline 6 & 74.36 & 83.94 & $15.53 \%$ \\
\hline 7 & 69.70 & 83.05 & $19.91 \%$ \\
\hline 8 & 65.78 & 79.57 & $20.96 \%$ \\
\hline 9 & 60.53 & 77.56 & $28.18 \%$ \\
\hline 10 & 56.41 & 74.51 & $32.08 \%$ \\
\hline
\end{tabular}

est for the depths ranging from $1 \mathrm{~mm}$ to $\sim 20$ $\mathrm{mm}$.

In the present study, the performance of PBC algorithms was compared with MC Code FLUKA. Overestimation of the range 300\%$2 \%$ in $0.2 \mathrm{gm} / \mathrm{cm}^{3}$ and $200 \%-1 \%$ in $0.4 \mathrm{gm} /$ $\mathrm{cm}^{3}$ density medium was produced by PBC algorithm compared to FLUKA MC Code [19].

As reported in earlier studies, $\mathrm{PBC}$ performs in poor way in case of lung tumor. PBC calculates dose taking a ray from the source of beam. To incorporate the density variations, each beam undergoes inhomogeneity corrections; however, the laxity of adjacent beams in dose deposition produces inaccurate results [20].

It was observed from the results that deviation was higher in $0.2 \mathrm{gm} / \mathrm{cm}^{3}$ compared to $0.4 \mathrm{gm} / \mathrm{cm}^{3}$ (Figure 8). The deviation in PBC calculated PDD was found in deeper point of interests as well as in depth of maximum dose (Dmax). The PBC calculated Dmax for $0.2 \mathrm{gm} / \mathrm{cm}^{3}$ and $0.4 \mathrm{gm} / \mathrm{cm}^{3}$ were $6 \mathrm{~mm}$ and $5 \mathrm{~mm}$, whereas FLUKA produced $10 \mathrm{~mm}$ and $18 \mathrm{~mm}$, respectively (Table 4). Such a type of 


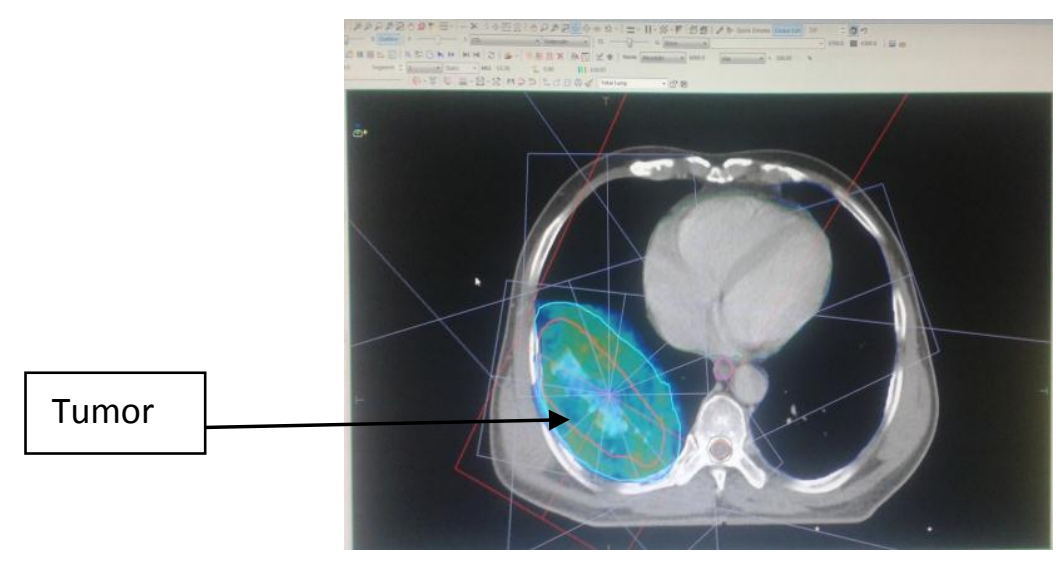

Figure 9: Transverse section of Thorax with tumor (contoured)

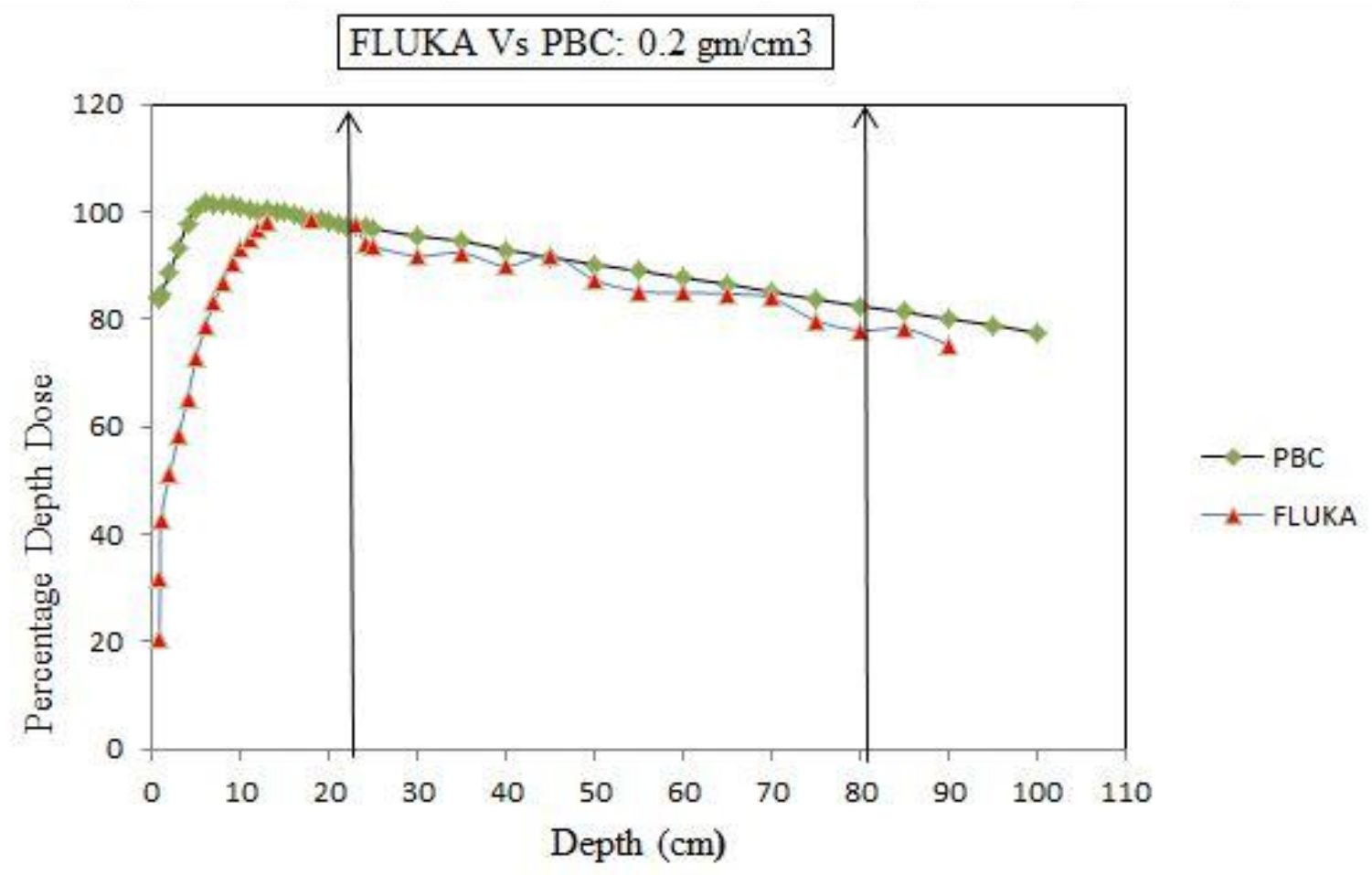

Figure 10: Variation of PDD with Depth calculated by FLUKA code and PBC for medium density $0.2 \mathrm{gm} / \mathrm{cm}^{3}$.

variation can be favorable in clinical situations like tumor lying at the same depth equal to Dmax, and this can be favorable in delivering lesser dose to normal structure with maximum dose to tumor. However, this can result in under dosing of tumors such as that found just beyond the inhomogeneous surface along the beam (Figure 9). The performance (PDD) of both PBC and FLUKA code was found almost the same over the depth ranging from $2.5 \mathrm{~cm}$ to $7 \mathrm{~cm}$ in case of density $0.4 \mathrm{gm} / \mathrm{cm}^{3}$; however, some deviations were recorded for $0.2 \mathrm{gm} /$ $\mathrm{cm}^{3}$.

The present study contains the analysis of 


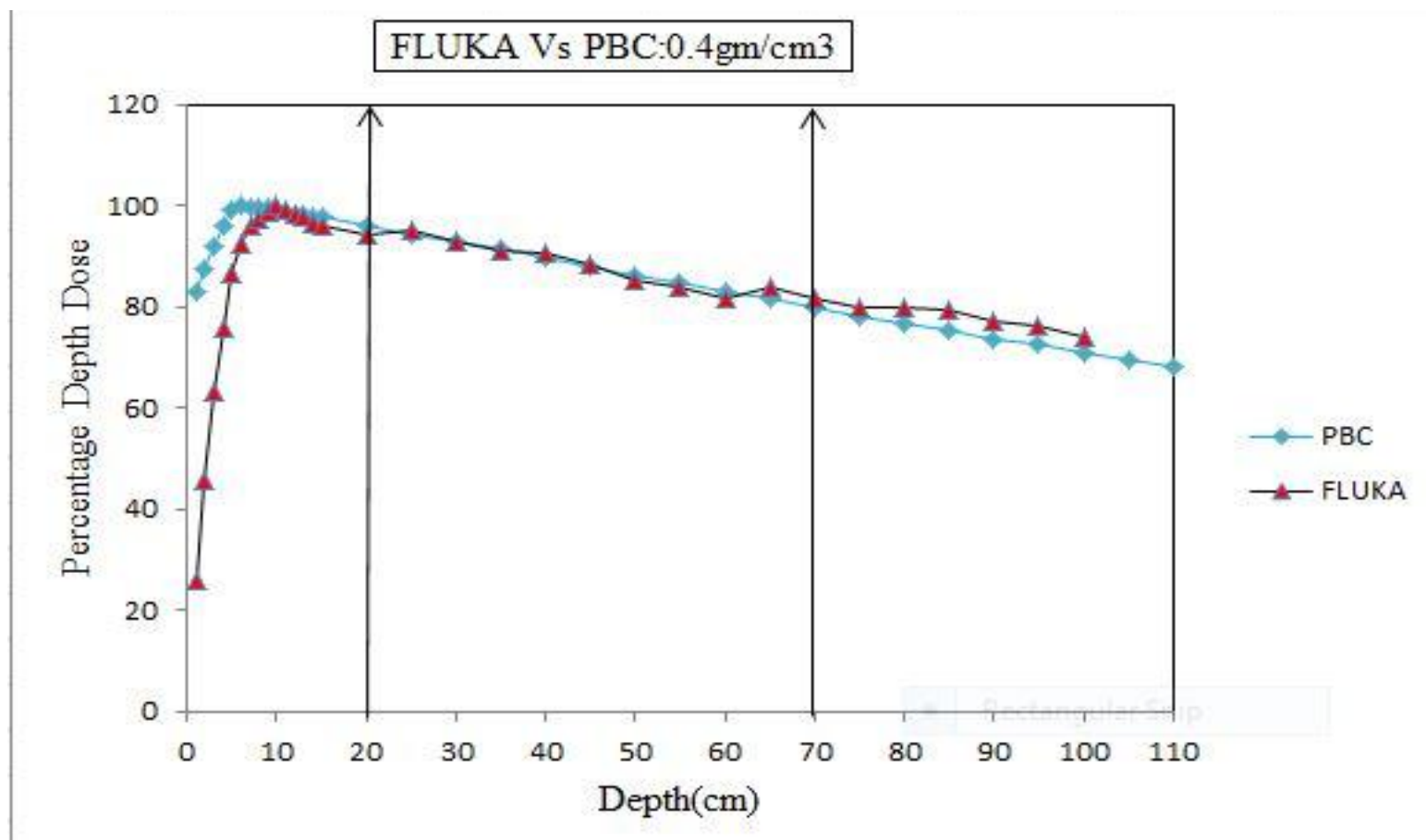

Figure 11: Variation of PDD with Depth calculated by FLUKA code and PBC for medium density $0.40 \mathrm{gm} / \mathrm{cm}^{3}$.

Table 4: Depth of Dmax for (Co-60) for different lung densities.

\begin{tabular}{cccc}
\hline Medium & Water & $\mathbf{0 . 4} \mathbf{~ g m} / \mathbf{c m}^{\mathbf{3}}$ & $\mathbf{0 . 2} \mathbf{~ g m} / \mathbf{c m}^{\mathbf{3}}$ \\
\hline $\begin{array}{c}\text { Depth } \\
\text { of } \text { Dmax } \\
(\mathbf{m m})\end{array}$ & 5 & 10 & 18 \\
\hline
\end{tabular}

single anterior beam using PBC algorithm. Large differences between planned and delivered dose have been recorded in case of sophisticated treatment planning systems too. Hideharu Miura et al. studied the impact of lung density and tumor position on lung stereotactic body radiotherapy taking a range of lung density and found reduction in dose to PTV with a decrease in lung density. A difference of more than $20 \%$ was found between planned and delivered dose due to the change in lung density [21].

The authors in the present study employed only Co-60 photon beam as source of irradiation; however, similar deviations have been recorded for other photon beam used in lung cancer radiotherapy. To assess the difference in planned and delivered dose due to change in lung density, Lasse Rye Aarup et al. created a virtual lung phantom having lung and spherical tumor inside the cubical body. The performance of different algorithms including PBC for 6 and $18 \mathrm{MV}$ beams was evaluated in terms of target coverage having lung density ranging from $0.1 \mathrm{gm} / \mathrm{cm}^{3}$ to $0.45 \mathrm{gm} / \mathrm{cm}^{3}$ and similar to the present study, increase in PBC overestimation in the target dose with decrease in lung density was found. In case of lung cancer radiotherapy, these variations in lung density need to be considered [22]. The overestimation by TPSs used in lung cancer radiotherapy basically results in under dosing to the target and which can be a cause behind higher recurrence and mortality rate in lung cancer [23].

\section{Conclusion}

Large variations in inter-lung \& intra-lung densities were observed. Lung density was found decreasing with age. In manual treatment time calculation for lung cancer radiotherapy, 
inhomogeneity correction factor should be used for a better dose delivery. Large overestimation in dose by PBC was found at air/lung interface as well as within the lung. The results of Monte Carlo simulation can be used to assess the performance of treatment planning systems used in lung cancer radiotherapy.

\section{Acknowledgment}

The authors are thankful to Mr. Hemant Kumar Patni of B.A.R.C., Mumbai, India for his help in Monte Carlo simulations with FLUKA code.

\section{Conflict of Interest}

None

\section{References}

1. Fu W, Dai J, Hu Y. The influence of lateral electronic disequilibrium on the radiation treatment planning for lung cancer irradiation. Biomed Mater Eng. 2004;14:123-6. PubMed PMID: 14757959.

2. Tada T, Minakuchi K, Sakamoto $H$, Fukuda $H$, Bun $\mathrm{M}$, Nakajima T. Inhomogeneity correction in radiotherapy for lung cancer in multicenter clinical trials. Radiat Med. 2002;20:191-4. PubMed PMID: 12296435.

3. Thomas SJ. A modified power-law formula for inhomogeneity corrections in beams of highenergy x rays. Med Phys. 1991;18:719-23. doi. org/10.1118/1.596665. PubMed PMID: 1921876.

4. Orton CG, Chungbin S, Klein EE, Gillin MT, Schultheiss TE, Sause WT. Study of lung density corrections in a clinical trial (RTOG 88-08). Radiation Therapy Oncology Group. Int $J$ Radiat Oncol Biol Phys. 1998;41:787-94. doi.org/10.1016/S03603016(98)00117-5. PubMed PMID: 9652839.

5. Papanikolaou N, Battista JJ, Boyer AL, Kappas C, Klein E, Mackie TR, et al. Tissue inhomogeneity corrections for megavoltage photon beams. AAPM Task Group. 2004;65:1-142.

6. Abdul Haneefa K, Cyriac TS, Musthafa M, Ganapathi Raman $R$, Hridya $V$, Siddhartha $A$, et al. FLUKA Monte Carlo for Basic Dosimetric Studies of Dual Energy Medical Linear Accelerator. Journal of Radiotherapy. 2014;2014.

7. Battistoni G, Cerutti F, Fasso A, Ferrari A, Muraro S, Ranft J, et al., editors. The FLUKA code: Description and benchmarking. Hadronic Shower Simulation Workshop (AIP Conference Proceedings Vol- ume 896); 2007.

8. Ferrari A, Sala PR, Fasso A, Ranft J. FLUKA: A multi-particle transport code (Program version 2005). 2005.

9. Botta F, Mairani A, Hobbs RF, Vergara Gil A, Pacilio M, Parodi K, et al. Use of the FLUKA Monte Carlo code for 3D patient-specific dosimetry on PET-CT and SPECT-CT images. Phys Med Biol. 2013;58:8099-120. doi.org/10.1088/00319155/58/22/8099. PubMed PMID: 24200697. PubMed PMCID: 4037810.

10. Taleei R, Shahriari M, AGHAMIRI SMR. Evaluation of FLUKA Code in Simulation and Design of X-ray Tubes for X-ray Profile. 2006.

11. Thomas SJ. Relative electron density calibration of CT scanners for radiotherapy treatment planning. Br J Radiol. 1999;72:781-6. doi.org/10.1259/ bjr.72.860.10624344. PubMed PMID: 10624344.

12. McKenzie A. Cobalt-60 gamma-ray beams. $B J R$ supplement/BIR. 1995;25:46-61.

13. Speigel M. Probability and Statistics. Schaum's Outline Series in Mathematics. McGraw-Hill, New York; 1975

14. Fujisaki T, Kikuchi K, Saitoh H, Tohyama N, Myojoyama $A$, Osawa $A$, et al. Effects of density changes in the chest on lung stereotactic radiotherapy. Radiat Med. 2004;22:233-8. PubMed PMID: 15468943.

15. AC03205248 A. Absorbed dose determination in external beam radiotherapy: an international code of practice for dosimetry based on standards of absorbed dose to water: Internat. Atomic Energy Agency; 2000.

16. Praveenkumar RD, Santhosh KP, Augustine A. Estimation of inhomogenity correction factors for a Co60 beam using Monte Carlo simulation. J Cancer Res Ther. 2011;7:308-13. doi.org/10.4103/09731482.87030. PubMed PMID: 22044813.

17. Verhaegen F, Seuntjens J. Monte Carlo modelling of external radiotherapy photon beams. Phys Med Biol. 2003;48:R107-64. doi.org/10.1088/00319155/48/21/R01. PubMed PMID: 14653555.

18. Joshi CP, Darko J, Vidyasagar PB, Schreiner LJ. Dosimetry of interface region near closed air cavities for Co-60, $6 \mathrm{MV}$ and $15 \mathrm{MV}$ photon beams using Monte Carlo simulations. J Med Phys. 2010;35:7380. doi.org/10.4103/0971-6203.62197. PubMed PMID: 20589116. PubMed PMCID: 2884308.

19. Robinson D. Inhomogeneity correction and the analytic anisotropic algorithm. J Appl Clin Med Phys. 2008;9:2786. PubMed PMID: 18714283.

20. Tachibana M, Noguchi Y, Fukunaga J, Hirano N, 
Yoshidome S, Hirose T. Influence on dose calculation by difference of dose calculation algorithms in stereotactic lung irradiation: comparison of pencil beam convolution (inhomogeneity correction: batho power law) and analytical anisotropic algorithm. Nihon Hoshasen Gijutsu Gakkai Zasshi. 2009;65:1064-72. doi.org/10.6009/jjrt.65.1064. PubMed PMID: 19721315.

21. Miura H, Masai N, Oh R-J, Shiomi H, Yamada K, Usmani MN, et al. Dosimetric Impact of Tumor Position and Lung Density Variations in Lung Stereotactic Body Radiotherapy. International Journal of Medical Physics, Clinical Engineering and Radia- tion Oncology. 2014;2014

22. Aarup LR, Nahum AE, Zacharatou C, Juhler-Nottrup T, Knoos T, Nystrom H, et al. The effect of different lung densities on the accuracy of various radiotherapy dose calculation methods: implications for tumour coverage. Radiother Oncol. 2009;91:405-14. doi.org/10.1016/j.radonc.2009.01.008. PubMed PMID: 19297051.

23. Dela Cruz CS, Tanoue LT, Matthay RA. Lung cancer: epidemiology, etiology, and prevention. Clin Chest Med. 2011;32:605-44. doi.org/10.1016/j. ccm.2011.09.001. PubMed PMID: 22054876. PubMed PMCID: 3864624. 